\title{
Leveraging CAM to treat depression and anxiety
}

\section{Conventional medications and psychotherapy are still first-line treatments, but certain complementary and alternative strategies have value as adjunctive measures.}

A lmost $8 \%$ of Americans ages $\geq 12$ years have depression and $19.1 \%$ of Americans ages $\geq 18$ years have experienced an anxiety disorder in the past year. ${ }^{1,2}$ Furthermore, suicide, which can result from depression and anxiety, is the 10th leading cause of death in the United States, claiming about 40,000 to 49,000 lives per year since 2012 , with increasing yearly rates. ${ }^{3}$ While multiple conventional medication and therapy treatments are available, patients remain interested in complementary and alternative medicine (CAM) options. According to the National Center for Complementary and Integrative Health, more than $30 \%$ of American adults use CAM treatments. ${ }^{4}$

This article provides an overview of the evidence for commonly used CAM treatments for unipolar depression and anxiety in adults. It is designed to serve as a useful resource when patients are interested in looking beyond conventional medications.

\section{St. John's wort:}

\section{'Yes' for depression; 'no' for anxiety}

Hypericum perforatum, more commonly known as St. John's wort, is a widely used antidepressant, especially in Europe where it is prescribed, rather than offered over the counter as it is here. Its mechanism of action is not completely understood because its various constituents have different neuropharmacologic activities. ${ }^{5,6}$

A 2008 Cochrane review evaluated 29 randomized, double-blind studies $(\mathrm{N}=5489)$ that compared St. John's wort with placebo or standard antidepressants in the treatment of depression. ${ }^{7}$ St. John's wort was found to be superior to placebo and comparable to standard antidepressants. More recently, a 2017 meta-analysis of 27 studies $(\mathrm{N}=3808)$ had similar findings. ${ }^{8}$ In patients with mild-to-moderate depression, St. John's wart produced rates of remission that were comparable to those produced by selective serotonin reuptake inhibitors (SSRIs) but with a lower discontinuation rate.
Amanda E. Olagunju, DO; Heidi Gaddey, MD Department of Family Medicine, Langley Hospital, Langley Air Force Base, VA (Dr. Olagunju); Department of Family Medicine, David Grant Medical Center, Travis Air Force Base, CA (Dr. Gaddey)

\section{Đamanda.olagunju@gmail.} com

The authors reported no potential conflict of interest relevant to this article.

The views expressed in this article are those of the authors and do not necessarily reflect the official policy or position of the US government or any other agency, organization, employer, or company. 


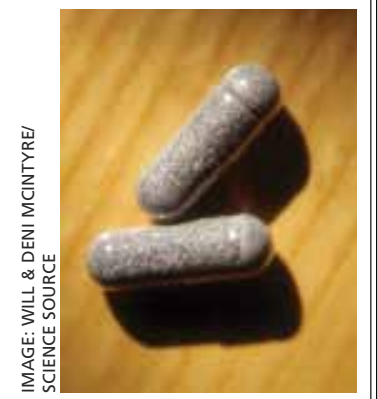

In patients with mildto-moderate depression, St. John's wort produced rates of remission that were comparable to those produced by SSRIswith a lower discontinuation rate.
I Mood disorders other than depression. Studies do not support a role for St. John's wort in the treatment of anxiety disorders. There are no trials that assess the efficacy of St. John's wort for the reduction of symptoms of general anxiety disorder as a primary outcome of treatment. Some small clinical trials have investigated the efficacy of St. John's wort in obsessive-compulsive disorder and social anxiety disorder. In those studies, St. John's wort performed no better than placebo., ${ }^{9,10}$

A few words of caution. Preparations of St. John's wort in the United States are not standardized, so St. John's wort should be used in America with caution. Furthermore, long-term use of St. John's wort for depression is questionable given that most studies have evaluated only up to 12 weeks of use. ${ }^{8}$

If used, studies indicate that the St. John's wort extract that should be used is $0.3 \%$ hypericin or $5 \%$ hyperforin administered in a dosage of 300 to $400 \mathrm{mg}$ tid. ${ }^{7,8,11}$ Physicians and patients can use www.consumerlabs. com to find St. John's wort brands that have met specified quality criteria based on independent laboratory studies. This Web site can also be used to investigate the quality of the brands available for the other supplements discussed in this article.

I Adverse effects. St. John's wort and an SSRI can lead to serotonin syndrome, which is a constellation of symptoms involving mental status changes and autonomic and neuromuscular hyperactivity caused by serotonin overactivity. ${ }^{12}$ Furthermore, treatment failures with anticoagulants, digoxin, hormonal contraceptives, immunosuppressants, and narcotics due to concomitant use with St. John's wort have been reported..$^{13}$

The most common adverse reactions to St. John's wort include gastrointestinal symptoms, dizziness, sedation, photosensitivity, dry mouth, urinary frequency, anorgasmia, and swelling. ${ }^{14}$ However, multiple studies have supported St. John's wort to be equally or better tolerated than conventional antidepressants. ${ }^{7,8}$

\section{Certain forms of folate can be} adjunctive treatment for depression

I Methylfolate is the form of folate that crosses the blood-brain barrier. A prospec- tive observational study evaluated the cerebral spinal fluid of 33 patients with refractory depression. The authors found metabolic abnormalities in the cerebrospinal fluid of most of those patients, the most common of which was folate deficiency in 12 patients despite normal serum folate levels. ${ }^{15}$

Additionally, current understanding of the role of the Methylenetetrahydrofolate reductase (MTHFR) gene and the folate cycle in depression supports a potential role of methylfolate in depression treatment. ${ }^{16}$ The MTHFR gene encodes for an enzyme called MTHFR. The MTHFR enzyme converts 5,10-MTHF to 5-MTHF, which then crosses the blood-brain barrier and donates a methyl group for the conversion of homocysteine to methionine. Methionine is a precursor to monoamine neurotransmitters. Thus, decreased expression of the MTHFR gene leads to decreased methylfolate levels, which, in turn, potentially leads to insufficient neurotransmitter synthesis and homocysteine excess.

MTHFR gene polymorphisms and increased homocysteine levels have been found to be associated with the occurrence of depression. One thought is that methylfolate supplementation compensates for an underlying MTHFR enzyme deficiency in patients with depression. Further studies are needed to determine if screening depressed patients for MTHFR gene polymorphisms is of benefit. ${ }^{16}$

A 2012 randomized controlled trial (RCT) $(\mathrm{N}=75)$ compared L-methylfolate $15 \mathrm{mg} / \mathrm{d}$ plus an SSRI with placebo plus an SSRI in patients with SSRI-resistant major depression. ${ }^{17}$ The trial found that a reduction of baseline symptoms by $\geq 50 \%$ occurred in more patients who received adjunctive L-methylfolate than placebo ( $32 \%$ vs $15 \%$ ) and tolerability was comparable. These findings were again supported in 2016 with a 12-month study showing L-methylfolate to have long-term tolerability comparable to placebo, ${ }^{18}$ and in 2017 with a randomized trial $(\mathrm{N}=260)$ that found escitalopram $10 \mathrm{mg} / \mathrm{d}$ plus L-methylfolate $15 \mathrm{mg} / \mathrm{d}$ to be significantly more effective at treating depression than escitalopram $10 \mathrm{mg} / \mathrm{d}$ alone. ${ }^{19}$ Thus, methylfolate may be an effective adjunc- 
tive treatment for depression at a dosage of $15 \mathrm{mg} / \mathrm{d}$.

- S-adenosyl methionine (SAMe) is a metabolite of folate derived from methionine that facilitates the synthesis of neurotransmitters including dopamine, norepinephrine, and serotonin. In Europe, as is the case with St. John's wort, it is a prescription medication.

A randomized trial $(\mathrm{N}=73)$ compared adjunctive SAMe $800 \mathrm{mg}$ bid with placebo in the treatment of patients with unipolar major depression who did not experience improvement with SSRI treatment alone. ${ }^{20}$ The investigators found that more patients who received SAMe than who received placebo had improvement in their depression $(36.1 \%$ vs $17.6 \%)$, and more patients who received SAMe compared to placebo went into depression remission (25.8\% vs $11.7 \%$ ).

Adverse effects were comparable in both groups. Thus, SAMe at a dosage of 400 to 1600 $\mathrm{mg} / \mathrm{d}$ may be effective in the treatment of depression. ${ }^{20,21}$ The findings of 1 study $(\mathrm{N}=65)$ suggest that patients could experience further improvement in their depression symptoms with SAMe at doses of as much as $\mathbf{3 2 0 0}$ $\mathrm{mg} / \mathrm{d}$; however, $3200 \mathrm{mg} / \mathrm{d}$ increased the occurrence of gastrointestinal adverse effects (31.3\% in the SAMe arm vs $3.8 \%$ in the placebo group). ${ }^{21}$

Folate. With regard to folate itself, randomized trials have not supported its efficacy in the treatment of depression in the general population. $^{22}$

\section{Vitamin D may improve anxiety/ depression in those with low levels} Vitamin D supplementation is also being used more frequently in the treatment of depression. Case-control, cross-sectional, and cohort studies have linked low vitamin D levels to the occurrence of depression. A 2013 systematic review of 14 such studies ( $\mathrm{N}=31,424)$ found lower vitamin D levels in people with depression compared with controls. ${ }^{23}$ Further studies are needed to determine if this relationship is causal, and quality RCTs investigating the effect of vitamin D supplementation on depression are lacking.
A 2019 study $(\mathrm{N}=30)$ evaluated the impact of vitamin D supplementation on generalized anxiety disorder in patients with cooccurring vitamin D deficiency. Half received standard-of-care general anxiety disorder treatment plus 50,000 IU of vitamin D weekly for 3 months, while the other half received standard of care alone. Significant improvements in anxiety scores, increases in serum serotonin, and decreases in serum neopterin (an inflammatory marker) were observed in the vitamin D-treated group compared to the group that did not receive vitamin D. ${ }^{24}$

It is not currently standard of care to check vitamin D levels in all patients presenting with mood disorders. However, if screening is indicated for another reason and low levels are confirmed, vitamin D replacement may improve anxiety and/or depressive symptoms. Despite this, no evidence exists to support vitamin D supplementation for depression or anxiety in patients with normal vitamin D levels. ${ }^{24,25}$

\section{The effects of omega-3 fatty acids are largely unclear}

Research has shown that omega-3 polyunsaturated fatty acids (n-3 PUFA), which are found in fish oil, protect glutamatergic neurotransmission from glucocorticoids, which are released in the body during a stress response. ${ }^{26}$ Small clinical trials have found n-3 PUFAs to reduce the symptoms of anxiety compared with placebo, but the acids have not been studied directly for anxiety disorders. ${ }^{27,28}$

With regard to depression, evidence is conflicting as to whether n-3 PUFAs are of any benefit in treatment. ${ }^{29}$ Eicosapentaenoic acid (EPA) and docosahexaenoic acid (DHA) are hypothesized to be the components in omega-3 fatty acid preparations that could lead to a reduction in depressive symptoms. However, randomized trials have shown that EPA-predominant omega-3 fatty acid formulations have only a moderate or no clinically significant effect on depression over placebo, and that DHA-predominant omega-3 fatty acid formulations are only comparable or inferior to placebo. ${ }^{30}$

Response to EPA may be greater in pa-

\section{S-adenosyl methionine (SAMe) at a dosage of 400 to $1600 \mathrm{mg} / \mathrm{d}$ may be effective in the treatment of depression.}




\section{$>$}

If screening for vitamin D levels is indicated for another reason and low levels are confirmed, vitamin D replacement may improve anxiety and/ or depression symptoms. tients with depression who have high levels of inflammatory biomarkers, such as interleuken (IL)-1 receptor antagonist (1Ra), IL-6, high-sensitivity C-reactive protein (hs-CRP), leptin, and adiponectin, than in patients with low levels. An 8-week trial randomly assigned patients with unipolar major depression $(\mathrm{N}=$ 155) to receive either EPA, DHA, or placebo and found that improvement for the 3 groups was comparable; however, in the subgroup of patients with high levels of inflammatory markers, improvement in depressive symptoms was significantly greater with EPA than with either DHA or placebo. ${ }^{31}$

It is unclear whether different sources of n-3 PUFA, such as whole fish vs fish oil vs prescription omega-3 acid ethyl esters (Lovaza), are more or less efficacious in the treatment of anxiety or depression. Furthermore, there is no standard dosing for n-3 PUFA in the treatment of mood disorders. Given that the US Food and Drug Administration recommends no more than $2 \mathrm{~g} / \mathrm{d}$ of combined EPA and DHA supplementation, we recommend using $2 \mathrm{~g} / \mathrm{d}$ if one decides to treat depression/ anxiety with n-3 PUFA. ${ }^{32}$

N-3 PUFA supplementation is fairly benign. There have been previous concerns about n-3 PUFA supplementation increasing patients' risk for gastrointestinal bleeding, but a 2006 systematic review that included 9 trials $(\mathrm{N}=2612)$ that looked at clinically significant bleeding episodes found that even patients at high risk for bleeding (ie, those taking aspirin or warfarin) had no increased bleeding risk from taking n-3 PUFA supplementation at up to $4 \mathrm{~g} / \mathrm{d}^{33}$

\section{Don't underestimate exercise and meditation; consider acupuncture}

I Exercise. Multiple practice guidelines, including the American Psychiatric Association's "Practice Guideline for the Treatment of Patients with Major Depressive Disorder," and meta-analyses have supported the use of exercise to treat unipolar major depression and anxiety. ${ }^{34-37}$ However, only about $26 \%$ of American men and 19\% of American women met the US Department of Health and Human Services' "Federal Physical Activity Guidelines for Americans" in 2016. ${ }^{38}$
Exercise alone is a reasonable monotherapy, as long as patients are monitored closely for worsening symptoms. Additionally, exercise as an add-on treatment can be helpful for more severe depression or anxiety. ${ }^{39}$ The best type, duration, and frequency of exercise specifically for the treatment of depression or anxiety has yet to be determined, but physicians may base their exercise recommendations on the "Federal Physical Activity Guidelines for Americans" for general good health (TABLE). ${ }^{38}$

I Meditation, especially mindfulness meditation, is another strategy that has gained popularity in the treatment of anxiety and depression. Mindfulness has been defined as "the practice of maintaining a nonjudgmental state of heightened or complete awareness of one's thoughts, emotions, or experiences on a moment-to-moment basis." ${ }^{40}$ A 2014 systematic review and meta-analysis of 47 trials with 3515 participants found that mindfulness meditation programs led to clinically significant moderate reductions in anxiety. ${ }^{41}$ Smaller effects were found for depression.

Nevertheless, meditation may be beneficial as an adjunctive treatment for depression. A small randomized trial $(\mathrm{N}=25)$ compared an adjunctive breathing-based meditation intervention with a waitlist control (delayed yoga) in patients with unipolar major depression who failed to respond to at least 8 weeks of antidepressant treatment. ${ }^{42}$ The meditation intervention consisted of a group program with sitting meditation, breathing exercises, and yoga postures. Participants engaged in the meditation intervention for 2 to 3.5 hours per day for 8 weeks and demonstrated significant improvement in depression symptoms compared with the control group. ${ }^{42}$

I Acupuncture. A 2018 meta-analysis of 64 studies $(\mathrm{N}=7104)$ suggests that acupuncture results in a small-to-moderate reduction in depressive symptoms when compared to no treatment, control/sham acupuncture, or medication. ${ }^{43}$ Furthermore, acupuncture plus medication compared to medication alone results in a higher reduction in depressive symptoms without an increase in adverse events. ${ }^{43}$

Additionally, a 2019 analysis of 10 systematic reviews found acupuncture to be 
TABLE

Federal Physical Activity Guidelines for Americans ${ }^{38}$

\begin{tabular}{l|l|l}
\hline & Frequency & Exertion goal \\
\hline Aerobic exercise & At least $150 \mathrm{~min} / \mathrm{wk}$ & Moderate $^{\text {a }}$ to vigorous intensity \\
\hline Resistance training & At least 2 sessions/wk & $\begin{array}{l}\text { Moderate }{ }^{\text {a }} \text { to vigorous intensity } ; \text { should involve all } \\
\text { major muscle groups }\end{array}$ \\
\hline
\end{tabular}

a Moderate-intensity exercise $=$ exercise requiring 3 to 6 metabolic equivalents of task (METs), such as walking briskly, raking the yard, gardening, or swimming leisurely.

${ }^{b}$ Vigorous-intensity exercise = exercise requiring $\geq 6$ METs, such as running, carrying heavy groceries upstairs, shoveling snow, or participating in a strenuous fitness class.

more effective than control/sham acupuncture in the treatment of general anxiety. ${ }^{44}$ It should be noted, however, that a lot of heterogeneity and potential for bias existed across all of the studies. The studies analyzed were very low to low in quality. Thus, the evidence is insufficient to strongly recommend the use of acupuncture for depression or anxiety, although acupuncture is a safe intervention with low rates of adverse events.

\section{Emotional support animals:}

\section{Beneficial, but evidence is weak}

Emotional support animals are gaining in popularity with Americans who have mood disorders. An important distinction must be made, however, between service animals and emotional support animals. A service animal is one "that is individually trained to do work or perform tasks for the benefit of an individual with a disability, including a physical, sensory, psychiatric, intellectual, or other mental disability." ${ }^{45}$ Under the Americans with Disabilities Act (ADA), service animals are limited to dogs, and, in some cases, specially trained miniature horses. Psychiatric service dogs can be trained to do anything from reminding their owner to take medicine to stopping self-mutilation activities.

Emotional support animals are not specially trained to perform tasks to help with disabilities. It's their companionship that helps relieve symptoms of depression and/ or anxiety. ${ }^{45}$ Thus, emotional support animals are not covered under federal laws that apply to service animals. However, the Air Carrier Access Act does require airlines to allow emotional support animals to fly in the cabin for free. Furthermore, the Fair Housing
Act allows emotional support animals to circumvent no-pet rules in housing and dorms. Airplanes and housing are the only places legally required to allow the unrestricted presence of emotional support animals. ${ }^{46}$

Also, there are important distinctions between emotional support animals and pets. While anyone can own a pet, an emotional support animal is prescribed by a licensed mental health professional as a treatment for a mood disorder. Housing facilities and airlines will usually require an emotional support animal "prescription" or letter from a physician to recognize animals as such.

A 2018 systematic review evaluated the evidence behind emotional support animals, which included 17 peer-reviewed journal articles, conference papers, and research dissertations $(\mathrm{N}=1727)$ mostly containing qualitative evidence. ${ }^{47}$ Unfortunately, there are no RCTs, and there are limited casecontrol and cohort studies evaluating the effect of an emotional support animal on mood disorders. Based on the available evidence, there does seem to be a psychological benefit to owning an animal for both those with a diagnosable mental health disorder and the general population. This benefit seems to stem from a perceived reduction in social isolation and an increase in emotional support. Factors that determine the psychological benefit of emotional support animals include the type of pet, the number of pets, the attachment to the pet, and the perceived friendliness of the pet. ${ }^{47}$

I Animal-assisted therapy. A 2014 systematic review evaluated higher-level evidence behind animal-assisted therapy (AAT) ${ }^{48}$ Although participating in therapy that involves interaction with animals is
Exercise alone is a reasonable monotherapy for depression or anxiety as long as patients are monitored closely for worsening symptoms. 


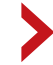

\section{Acupuncture plus medication compared to medication alone results in a higher reduction in depressive symptoms without an increase in adverse events.}

not the same as owning an emotional support animal, the concept-using an animal to improve mental health-is the same. The systematic review looked at 11 RCTs $(\mathrm{N}=$ 411) that studied the effect of AAT on mental health. Animals studied included dogs, cats, dolphins, birds, cows, rabbits, ferrets, and guinea pigs. Mental health disorders studied included schizophrenia, depression, anxiety, alcohol/drug abuse, and other addictive behaviors.

Therapeutic animal exposure led to reported improvements in mood, quality of life, and social behavior. These improvements were attributed to the animals buffering people's reactions to mental stressors. The animals provided a sense of comfort and safety and diverted attention away from immediate stressors. Furthermore, the memory of the animals brought participants a sense of comfort/happiness when they were later without the animal. However, the majority of participants were people who liked animals at baseline. ${ }^{48}$

\section{CORRESPONDENCE}

Amanda E. Olagunju, DO, Operational Medicine Clinic, Langley AFB Hospital, 77 Nealy Avenue, Langley AFB, VA 23665; amanda.olagunju@gmail.com.

\section{References}

1. National Center for Health Statistics, Centers for Disease Control and Prevention. FastStats: Depression. Last reviewed October 7, 2015. www.cdc.gov/nchs/fastats/depression.htm. Accessed May $26,2020$.

2. National Institute of Mental Health. Mental health information-statistics: any anxiety disorder. Last updated November 2017. www.nimh.nih.gov/health/statistics/any-anxiety-disorder. shtml. Accessed May 26, 2020.

3. Hedegaard H, Curtin SC, Warner M. Increase in suicide mortality in the United States, 1999-2018. NCHS Data Brief, no 362. Hyattsville, MD: National Center for Health Statistics; 2020.

4. National Center for Complementary and Integrative Health. Complementary, alternative, or integrative health: what's in a name? Last updated July 2018. www.nccih.nih.gov/health/ complementary-alternative-or-integrative-health-whats-in-aname. Accessed May 26, 2020.

5. Bennett DA Jr, Phun L, Polk JF, et al. Neuropharmacology of St. John's wort (Hypericum). Ann Pharmacother. 1998;32:1201-1208.

6. Müller WE, Singer A, Wonnemann M, et al. Hyperforin represents the neurotransmitter reuptake inhibiting constituent of hypericum extract. Pharmacopsychiatry. 1998;31(suppl 1):16-21.

7. Linde K, Berner MM, Kriston L. St John's wort for major depression. Cochrane Database Syst Rev. 2008;CD000448.

8. Ng QX, Venkatanarayanan N, Ho CY. Clinical use of Hypericum perforatum (St John's wort) in depression: a meta-analysis. J Affect Disord. 2017;210:211-221.

9. Kobak KA, Taylor LV, Bystritsky A, et al. St John's wort versus placebo in obsessive-compulsive disorder: results from a doubleblind study. Int Clin Psychopharmacol. 2005;20:299-304.

10. Kobak KA, Taylor LV, Warner G, et al. St. John's wort versus place- bo in social phobia: results from a placebo-controlled pilot study. J Clin Psychopharmacol. 2005;25:51-58.

11. Product reviews: St. John's wort supplements review. ConsumerLab.com. September 23, 2016. www.consumerlab.com/reviews/ St_Johns_Wort/stjohnswort/. Accessed May 26, 2020.

12. Simhan S. Serotonin syndrome. In: Abd-Elsayed A. (ed) Pain: A Review Guide. New York, NY: Springer; 2019.

13. Chrubasik-Hausmann S, Vlachojannis J, McLachlan A. Understanding drug interactions with St. John's wort (Hypericum perforatum L.): impact of hyperforin content. J Pharm Pharmacol. 2019;71:129-138.

14. Knüppel L, Linde K. Adverse effects of St. John's wort: a systematic review. JClin Psychiatry. 2004;65:1470-1479.

15. Pan LA, Martin P, Zimmer T, et al. Neurometabolic disorders: potentially treatable abnormalities in patients with treatmentrefractory depression and suicidal behavior. Am J Psychiatry. 2017; 174:42-50.

16. Kandler C, Lam S. Methylenetetrahydrofolate reductase screening in treatment-resistant depression. Fed Pract. 2019;36:207-208.

17. Papakostas GI, Shelton RC, Zajecka JM, et al. L-methylfolate as adjunctive therapy for SSRI-resistant major depression: results of two randomized, double-blind, parallel-sequential trials. $\mathrm{Am}$ JPsychiatry. 2012;169:1267-1274.

18. Zajecka J, Fava M, Shelton R, et al. Long-term efficacy, safety, and tolerability of L-methylfolate calcium $15 \mathrm{mg}$ as adjunctive therapy with selective serotonin reuptake inhibitors: a 12-month, openlabel study following a placebo-controlled acute study. JClin Psychiatry. 2016;77:654-660.

19. Kakar MS, Jehangir S, Mustafa M, et al. Therapeutic efficacy of combination therapy of L-methylfolate and escitalopram in depression. Pakistan Armed Forces Med J. 2017;67:976-981.

20. Papakostas GI, Mischoulon D, Shyu I, et al. S-adenosyl methionine (SAMe) augmentation of serotonin reuptake inhibitors for antidepressant nonresponders with major depressive disorder: a double-blind, randomized clinical trial. Am J Psychiatry. 2010;167:942-948.

21. Sakurai H, Carpenter L, Tyrka A, et al. Dose increase of S-adenosyl-methionine and escitalopram in a randomized clinical trial for major depressive disorder. J Affect Disord. 2020;262:118-125.

22. Sarris J, Murphy J, Mischoulon D, et al. Adjunctive nutraceuticals for depression: a systematic review and meta-analyses. Am J Psychiatry. 2016;173:575-587.

23. Anglin RE, Samaan Z, Walter SD, et al. Vitamin D deficiency and depression in adults: systematic review and meta-analysis. $\mathrm{Br} J$ Psychiatry. 2013;202:100-107.

24. Eid A, Khoja S, AlGhamdi S, et al. Vitamin D supplementation ameliorates severity of generalized anxiety disorder (GAD). Metab Brain Dis. 2019;34:1781-1786.

25. Li G, Mbuagbaw L, Samaan Z, et al. Efficacy of vitamin D supplementation in depression in adults: a systematic review. J Clin En docrinol Metab. 2014;99:757-767.

26. Hennebelle M, Champeil-Potokar G, Lavialle M, et al. Omega-3 polyunsaturated fatty acids and chronic stress-induced modulations of glutamatergic neurotransmission in the hippocampus. Nutr Rev. 2014;72:99-112.

27. Kiecolt-Glaser JK, Belury MA, Andridge R, et al. Omega-3 supplementation lowers inflammation and anxiety in medical students: a randomized controlled trial. Brain Behav Immun. 2011;25:17251734.

28. Buydens-Branchey L, Branchey M, Hibbeln JR. Associations between increases in plasma $n-3$ polyunsaturated fatty acids following supplementation and decreases in anger and anxiety in substance abusers. Prog Neuropsychopharmacol Biol Psychiatry. 2008;32:568-575.

29. Lin PY, Mischoulon D, Freeman MP, et al. Are omega-3 fatty acids antidepressants or just mood-improving agents? The effect depends upon diagnosis, supplement preparation, and severity of depression. Mol Psychiatry 2012;17:1161-1163.

30. Hallahan B, Ryan T, Hibbeln JR, et al. Efficacy of omega-3 highly unsaturated fatty acids in the treatment of depression. $\mathrm{Br} J$ Psychiatry. 2016;209:192-201.

31. Rapaport MH, Nierenberg AA, Schettler PJ, et al. Inflammation as a predictive biomarker for response to omega-3 fatty acids in major depressive disorder: a proof-of-concept study. Mol Psychiatry. 2016;21:71-79.

32. National Institutes of Health Office of Dietary Supplements. Omega-3 fatty acids. Updated October 17, 2019. https://ods. od.nih.gov/factsheets/Omega3FattyAcids-HealthProfessional. Accessed May 26, 2020. 
33. Wang C, Harris WS, Chung M, et al. $n-3$ fatty acids from fish or fish-oil supplements, but not alpha-linoleic acid, benefit cardiovascular disease outcomes in primary- and secondary- prevention studies: a systematic review. Am J Clin Nutr. 2006;84:5-17.

34. American Psychiatric Association Practice. Guideline for the Treatment of Patients with Major Depressive Disorder. 3rd Edition. 2010. https://psychiatryonline.org/pb/assets/raw/sitewide/ practice_guidelines/guidelines/mdd.pdf. Accessed May 26, 2020

35. Gordon B, McDowell C, Lyons M, et al. The effects of resistance exercise training on anxiety: a meta-analysis and metaregression analysis of randomized controlled trials. Sports Med. 2017;47:2521-2532.

36. Stubbs B, Vancampfort D, Rosenbaum S, et al. An examination of the anxiolytic effects of exercise for people with anxiety and stress-related disorders: a meta-analysis. Psychiatry Res. 2017;249:102-108.

37. Rethorst CD, Trivedi MH. Evidence-based recommendations for the prescription of exercise for major depressive disorder. $J$ Psychiatr Pract. 2013;19:204-212.

38. US Department of Health and Human Services. Physical Activity Guidelines for Americans. 2nd edition. Washington, DC: US Department of Health and Human Services; 2018.

39. Cooney GM, Dwan K, Greig CA, et al. Exercise for depression. Cochrane Database Syst Rev. 2013;CD004366.

40. Merriam-Webster Dictionary. "Mindfulness." www.merriamwebster.com/dictionary/mindfulness. Accessed May 26, 2020.
41. Goyal M, Singh S, Sibinga EM, et al. Meditation programs for psychological stress and well-being: a systematic review and metaanalysis. JAMA Intern Med. 2014;174:357-368.

42. Sharma A, Barrett MS, Cucchiara AJ, et al. A breathing-based meditation intervention for patients with major depressive disorder following inadequate response to antidepressants: a randomized pilot study. J Clin Psychiatry. 2017;78:e59-e63.

43. Smith CA, Armour M, Soo Lee M, et al. Acupuncture for depression. Cochrane Database Syst Rev. 2018;CD004046.

44. Li M, Xing X, Yao L, et al. Acupuncture for treatment of anxiety, an overview of systematic reviews. Complement Ther Med. 2019;43:247-252.

45. Brennan J. Service animals and emotional support animals. ADA National Network Information Guidance and Training on the Americans with Disabilities Act. Last updated April 2020. adata. $\mathrm{org} /$ publication/service-animals-booklet. Accessed May 26, 2020.

46. Clay RA. Is that a pet or therapeutic aid? Monitor on Psychology. 2016;47:38.

47. Brooks HL, Rushton K, Lovell K, et al. The power of support from companion animals for people living with mental health problems: a systematic review and narrative synthesis of the evidence. BMC Psychiatry. 2018;18:31.

48. Kamioka $\mathrm{H}$, Okada $\mathrm{S}$, Tsutani $\mathrm{K}$, et al. Effectiveness of animalassisted therapy: a systematic review of randomized controlled trials. Complement Ther Med. 2014;22:371-390.

A special supplement to The Journal of Family Practice ${ }^{\circledR}$

\section{Hot Topics in Primary Care}

Discussion of primary care topics includes expert insight into:
- Heart Failure in Type 2 Diabetes
- Naproxen vs Opioids
- Statin Selection
- Cluster Headache

\section{FREE 4 CME CREDITS}
- Insomnia in Alzheimer's Disease
- Diabetes Management
- Chronic Kidney Disease in Type 2 Diabetes
- Irritable Bowel Syndrome

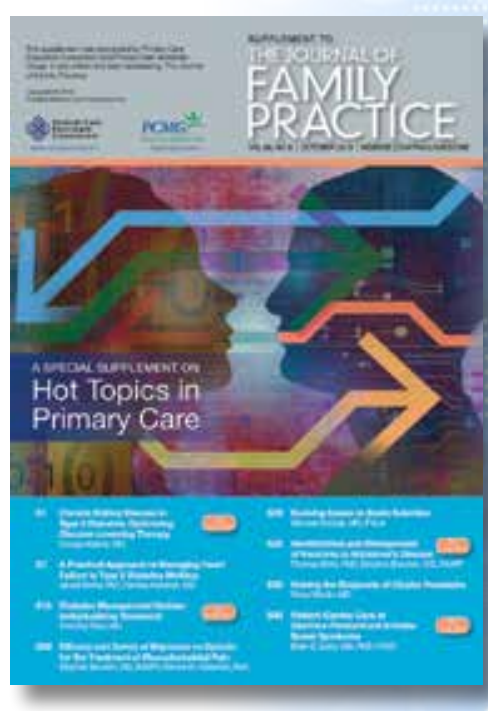

This supplement can be found at www.mdedge.com/familymedicine or directly at www.mdedge.com/hottopics2019 